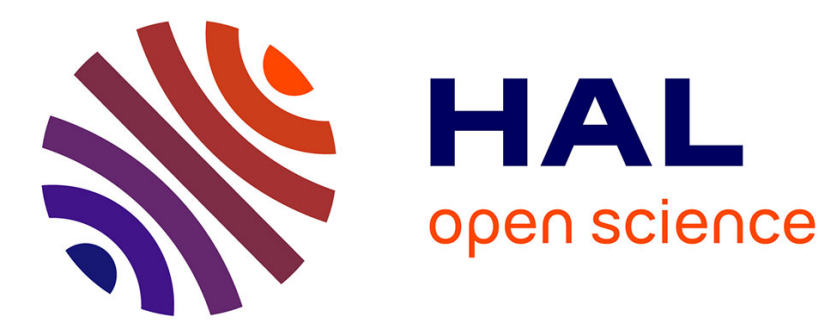

\title{
Beam Loading by Distributed Injection of Electrons in a Plasma Wakefield Accelerator
}

N Vafaei-Najafabadi, K A Marsh, C E Clayton, W An, W B Mori, C Joshi, W Lu, E Adli, Sébastien Corde, M Litos, et al.

\section{- To cite this version:}

N Vafaei-Najafabadi, K A Marsh, C E Clayton, W An, W B Mori, et al.. Beam Loading by Distributed Injection of Electrons in a Plasma Wakefield Accelerator. Physical Review Letters, 2014, 112, pp.025001. 10.1103/PhysRevLett.112.025001 . hal-01172417

\section{HAL Id: hal-01172417 \\ https://hal.science/hal-01172417}

Submitted on 7 Jul 2015

HAL is a multi-disciplinary open access archive for the deposit and dissemination of scientific research documents, whether they are published or not. The documents may come from teaching and research institutions in France or abroad, or from public or private research centers.
L'archive ouverte pluridisciplinaire HAL, est destinée au dépôt et à la diffusion de documents scientifiques de niveau recherche, publiés ou non, émanant des établissements d'enseignement et de recherche français ou étrangers, des laboratoires publics ou privés. 


\title{
Beam Loading by Distributed Injection of Electrons in a Plasma Wakefield Accelerator
}

\author{
N. Vafaei-Najafabadi, ${ }^{1}$ K. A. Marsh, ${ }^{1}$ C. E. Clayton, ${ }^{1}$ W. An, ${ }^{1}$ W. B. Mori, ${ }^{1,2}$ C. Joshi, ${ }^{1,}{ }^{*}$ W. Lu, ${ }^{3,2}$ E. Adli, ${ }^{4,5}$ \\ S. Corde, ${ }^{4}$ M. Litos, ${ }^{4}$ S. Li,${ }^{4}$ S. Gessner, ${ }^{4}$ J. Frederico, ${ }^{4}$ A. S. Fisher ${ }^{4}$ Z. Wu,${ }^{4}$ D. Walz,${ }^{4}$ R. J. England, ${ }^{4}$ \\ J. P. Delahaye, ${ }^{4}$ C. I. Clarke, ${ }^{4}$ M. J. Hogan, ${ }^{4}$ and P. Muggli ${ }^{6}$ \\ ${ }^{1}$ Department of Electrical Engineering, University of California Los Angeles, Los Angeles, California 90095, USA \\ ${ }^{2}$ Department of Physics and astronomy, University of California Los Angeles, Los Angeles, California 90095, USA \\ ${ }^{3}$ Department of Engineering Physics, Tsinghua University, Beijing 100084, China \\ ${ }^{4}$ SLAC National Accelerator Laboratory, Menlo Park, California 94025, USA \\ ${ }^{5}$ Department of Physics, University of Oslo, 0316 Oslo, Norway \\ ${ }^{6}$ Max Planck Institute for Physics, 80805 Munich, Germany
}

(Received 17 May 2013; published 15 January 2014)

\begin{abstract}
We show through experiments and supporting simulations that propagation of a highly relativistic and dense electron bunch through a plasma can lead to distributed injection of electrons, which depletes the accelerating field, i.e., beam loads the wake. The source of the injected electrons is ionization of the second electron of rubidium ( $\mathrm{Rb}$ II) within the wake. This injection of excess charge is large enough to severely beam load the wake, and thereby reduce the transformer ratio $T$. The reduction of the average $T$ with increasing beam loading is quantified for the first time by measuring the ratio of peak energy gain and loss of electrons while changing the beam emittance. Simulations show that beam loading by Rb II electrons contributes to the reduction of the peak accelerating field from its weakly loaded value of $43 \mathrm{GV} / \mathrm{m}$ to a strongly loaded value of $26 \mathrm{GV} / \mathrm{m}$.
\end{abstract}

DOI: 10.1103/PhysRevLett.112.025001

PACS numbers: 52.59.-f, 52.25.Jm, 52.35.Mw, 52.70.Nc

The FACET facility [1] at the SLAC National Accelerator Laboratory is presently being used for basic research on the plasma wakefield accelerator (PWFA). In this concept, a short, dense electron bunch [2] is used to generate a high gradient accelerating field in plasma that accelerates a second trailing beam to high energies [3]. Two critical physics issues that affect the energy gain and the emittance of the accelerated beam are beam head erosion [4] and ion motion [5]. The severity of both effects can be mitigated by using a plasma containing a heavier ion species such as rubidium $(\mathrm{Rb})$, rather than the previously used lithium $[6,7]$. In this Letter, we show that the maximum accelerating gradient that a wake can support is determined not by the plasma density as expected [8], but by the onset of electron injection due to further ionization of rubidium ions. This injected charge extracts energy from the wake in a process known as beam loading and thereby reduces the maximum energy gained by the electrons. Other heavier ions that are potential candidates [9] for mitigating the aforementioned issues may have a similar limitation on the maximum achievable acceleration gradient.

The onset of electron injection in the wake leads to a reduction in the important figure of merit for a PWFA known as the transformer ratio $T=E^{+} / E^{-}$[10]. Here, $E^{-}$and $E^{+}$are the peak decelerating and accelerating fields that act upon the beam electrons, respectively. In this experiment, injected electrons are generated via the further ionization of $\mathrm{Rb}^{+}$by the beam electrons' self-field or induced wakefield. These "Rb II electrons," observed as excess charge exiting the plasma, are born within the wake [11-14] as $\mathrm{Rb}^{+}$is ionized to $\mathrm{Rb}^{2+}$ at the tightly pinched regions of the initially mismatched drive bunch as it executes envelope oscillations [15] in the plasma. Since different slices of the beam lose energy at a different rate due to the wakefield, betatron oscillations of these beam slices quickly become out of phase resulting in continuous injection of the Rb II electrons. This unwanted beam loading results in a reduced average transformer ratio $\langle T\rangle$. Simulations confirm that, at a certain location in the wake, $T$ is reduced from a value of $1.4\left(E^{+}=43 \mathrm{GV} / \mathrm{m}\right)$ to about $1\left(E^{+}=26 \mathrm{GV} / \mathrm{m}\right)$, mainly because of this strong beam loading effect.

For plasma wakes in the blowout regime, where the electron beam density $n_{b}$ is larger than the plasma density, $n_{p}$ [16], significant beam loading will occur [17] when the loaded charge $Q(\mathrm{nC})>\left[\left(0.047 m c \omega_{p}\right) / e E_{s}\right]$ $\sqrt{10^{16} / n_{p}\left(\mathrm{~cm}^{-3}\right)}\left(k_{p} R_{b}\right)^{4}$. Here, we take $e E_{s} / m c \omega_{p}=$ $k_{p} R_{b} / 2$ as the electric field seen by the injected electrons, $k_{p}^{-1}$ is the plasma skin depth, and $R_{b}$ is the blowout radius of the wake. Thus, for the parameters of this experiment, $n_{p}=2.7 \times 10^{17} \mathrm{~cm}^{-3}, N=1.3 \times 10^{10}, \sigma_{r}^{*}=3 \mu \mathrm{m}$, $\sigma_{z}=40 \mu \mathrm{m}$; beam loading is expected to become significant if $Q>100 \mathrm{pC}$ or $6 \times 10^{8}$ electrons. Here, $N$ is the total number of particles that actually participate in the wake production [18] in the bi-Gaussian beam with a peak density of $n_{b}=N /(2 \pi)^{1.5} \sigma_{r}^{* 2} \sigma_{z}$, where $\sigma_{r}^{*}$ and $\sigma_{z}$ are the minimum bunch radius inside the plasma and bunch length, 
respectively. As the charge contained in the beam load approaches this value, $E^{+}$will be reduced compared to its unloaded value. This implies that the maximum energy gained by the accelerating electrons will be reduced in the presence of significant, unwanted charge injection.

In previous PWFA experiments [3], it has been shown that "beam-head erosion" limits the energy gain of electrons when the beam's electric field itself is used to form the plasma. Briefly, the $100 \%$ ionization contour of the plasma continuously recedes backwards in the beam frame as the front of the beam slowly expands, until it no longer is able to excite an intense wakefield. The speed at which the ionization contour erodes backwards scales [19] as ionization potential (IP) to the power 1.73. This is because atoms of a gas that have a lower ionization potential will be ionized earlier during the rising charge density of the beam, and thus, the beam will propagate further before it completely "diffracts" away due to the beam emittance. Thus, the $20.3 \mathrm{GeV}$ electron beam is expected to propagate $40 \%$ further (and, therefore, give a correspondingly higher energy gain) in a self-ionized $\mathrm{Rb}$ (ionization potential IP $4.4 \mathrm{eV}$ ) plasma, compared to the previously used $\mathrm{Li}$ (IP $5.1 \mathrm{eV}$ ) plasma.

A second motivation for exploring $\mathrm{Rb}$ is the need to mitigate emittance dilution of the electron beam due to possible ion motion [5] in future applications. In the "blow-out" regime [16] referred to earlier, the plasma electrons are completely blown out, leaving behind a region that contains the more massive, less mobile ions. This ion column exerts a linear focusing force on the electron beam, which preserves the transverse phase space of a matched beam [7], i.e., with $\sigma_{r}^{2}=\epsilon_{n}\left(c / \omega_{p}\right) \sqrt{2 / \gamma}$. If the density of the beam is large enough - $\left(n_{b} / n_{p}\right)>m_{i} / m_{e}$ - the ions are attracted toward the electron beam axis and the focusing force of the resulting ion column is no longer linear, which will, in turn, lead to the growth of the normalized emittance $\epsilon_{n}$ [5]. Since the extent of the ion motion scales inversely with the mass of the ions, heavier $\mathrm{Rb}$ atoms are preferred over the previously used $\mathrm{Li}$ atoms.

The experiments were carried out at the FACET facility with the setup shown in Fig. 1. The $20.3 \mathrm{GeV}$ electron bunch, containing $1.8 \times 10^{10}$ particles with $\sigma_{z} \sim 35-50 \mu \mathrm{m}$ [20] was focused to a vacuum $\sigma_{r}$ of $35 \mu \mathrm{m}$ at the beginning of the plateau region (i.e., after the up ramp, described below) of the $\mathrm{Rb}$ vapor in a heat pipe oven [21]. The electrons within the bunch were used to ionize $\mathrm{Rb}$, excite a wake, and probe it [6].

The rubidium vapor was collisionally contained in the hot region of the oven by an argon buffer gas. The rubidium density profile can be approximated by a trapezoidal shape with $10 \mathrm{~cm}$ up ramp, $20 \mathrm{~cm}$ plateau region, and $10 \mathrm{~cm}$ down ramp. In the $10 \mathrm{~cm}$ long boundary region on either side of the heated region, the density of argon must rise as the rubidium density falls to maintain pressure balance. In the

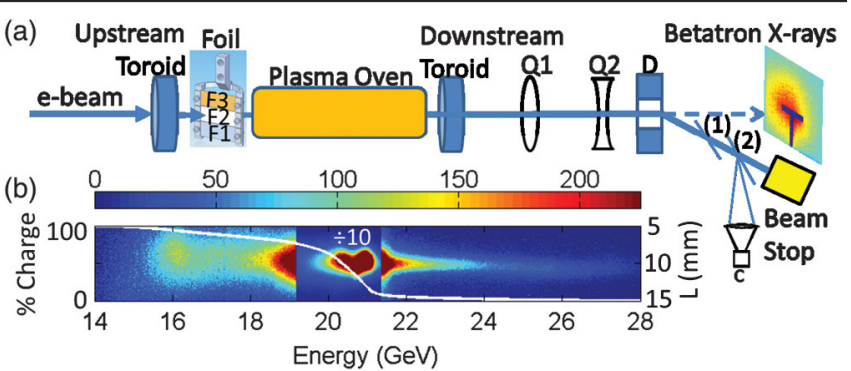

FIG. 1 (color). (a) The experimental setup. The dipole (D) and quadrapole magnets (Q1 and Q2) form the imaging spectrometer used as the energy diagnostic, Cherenkov radiation produced in air between $\mathrm{Si}$ wafers (1) and (2) is recorded using a 12 bit camera (C). Foils can be inserted in the beam (arrow) as it enters the plasma to change $\epsilon_{N}$ by factors of $1.2(F 1), 1.3(F 2)$, and 1.4 $(F 3)$, respectively, from the nominal value of $\epsilon_{N 0}=250 \times 50 \mu \mathrm{m}$ without any foils. The Lanex screen, placed behind a $1 \mathrm{~mm}$ copper sheet, is the betatron x-ray diagnostic; (b) an example of the energy spectrum with the white curve showing the percentage of the total beam charge. The charge near $21 \mathrm{GeV}$, observed not to lose any energy, has been attenuated by a factor of 10 on the image. The electron bunch has a head-to-tail correlated energy spread of $0.5 \mathrm{GeV}$.

central $20 \mathrm{~cm}$ long heated region, the $\mathrm{Rb}$ density is nearly constant at $2.7 \times 10^{17} \mathrm{~cm}^{-3}$.

The total charge that enters and leaves the heat pipe oven is measured using two absolutely calibrated toroids. The downstream toroid measures the beam charge plus any accelerated excess charge $(\Delta Q)$ from further ionization of $\mathrm{Rb}$ and Ar. Second, the dispersed spectrum of the beam electrons is recorded by camera $C$, an example of which is shown in Fig. 1(b). The spectra are first summed in the nondispersed direction and then integrated along the dispersed direction. The running integral is shown as the white curve in Fig. 1(b) which is used to define the maximumenergy ( $2 \%$ of the total charge) and the minimum energy (98\% of the total charge) of the electrons. We use these levels to calculate $\langle T\rangle \equiv \Delta W^{+} / \Delta W^{-}=\int E^{+} d z / \int E^{-} d z$, taking into account the initial head-to-tail correlated energy spread of the electrons. Since the electron bunch is ultrarelativistic, there is no relative motion between the particles, so the change in energy of any longitudinal slice of the beam is equal to the axially integrated electric field at the location of that slice over the length of the interaction.

As the bunch enters the up-ramp region, the electric field of the rising edge of the bunch easily ionizes the $\mathrm{Rb}$ atoms to $\mathrm{Rb}^{+}$and begins to blow out the plasma electrons. The experiment rapidly evolves into the blow-out regime as the focusing force of the ions pinches the initially $\sigma_{r}=35 \mu \mathrm{m}$ beam to $\sigma_{r}^{*}=3 \mu \mathrm{m}$, after which its envelope oscillates along the entire length of the ion column [11]. These envelope oscillations have a calculated wavelength of about $0.9 \mathrm{~cm}$ based on the experimental parameters. If $\sigma_{r}^{*}$ is small enough, the combined action of the beam's radial field $E_{r}$ and the wakefield $E_{z}$ can ionize $\mathrm{Rb}^{+}$to $\mathrm{Rb}^{2+}$. The 
threshold electric field (defined here as $10 \%$ ionization level) needed to produce $\mathrm{Rb}^{2+}(\mathrm{IP}=27.3 \mathrm{eV})$ is calculated using the Ammosov-Delone-Krainov (ADK) model [22] as $53 \mathrm{GV} / \mathrm{m}$. Taking $\sigma_{r}^{*}$ of $3 \mu \mathrm{m}$, we can estimate the maximum $E_{r}$ at the peak of the bi-Gaussian beam to be $E_{r}^{\max }=17.3 \mathrm{GV} / \mathrm{m}\left(N / 10^{10}\right)\left(10 \mu \mathrm{m} / \sigma_{r}^{*}\right)\left(30 \mu \mathrm{m} / \sigma_{z}\right)=$ $56 \mathrm{GV} / \mathrm{m}$. Thus, the electron bunch produces $\mathrm{Rb}$ II electrons along the length of the plasma at the minimum of each slice of the beam as it executes envelope oscillations. Since the bunch loses energy in the decelerating portion of the wake along this length, we expect there to be a strong correlation between the energy loss and the excess charge in the experiment. Note that, although the beam envelope oscillations could in principle be eliminated by matching the beam to the plasma, the envelope radius of the matched beam has to be large enough so that the combined field of the electron beam and the wake does not exceed the ionization threshold of the rubidium.

Distributed production of dark current is demonstrated from the correlation of $\Delta Q$ with the energy loss of the electron bunch, which increases as the bunch propagates further into the plasma. This distance, which can be less than the oven length, may be modified by varying either the beam emittance $\epsilon_{N}$ or the beam current. Both factors affect the head erosion rate, which is proportional to $\epsilon_{N}$, and $I^{-3 / 2}$ [4], where $I$ is the peak beam current $\propto 1 / \sigma_{z}$. We first vary $\epsilon_{N}$ (by inserting foils $F 1, F 2$, and $F 3$ in the beam path) while keeping $\sigma_{z}$ and, therefore, the current profile of the beam constant. From Fig. 2(a), one can see that as the beam $\epsilon_{N}$ is decreased, $\Delta W^{-}$and the excess charge $\Delta Q$ increase. This is because a decrease in $\epsilon_{N}$ decreases the head erosion rate, thereby increasing the bunch propagation distance (and, therefore, $\Delta W^{-}$) in the plasma. The increase in $\Delta Q$

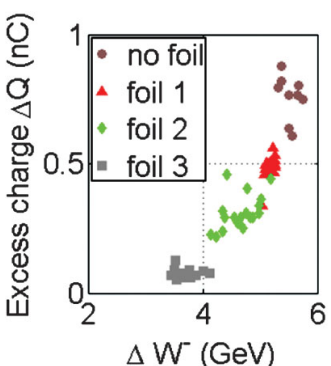

(a)

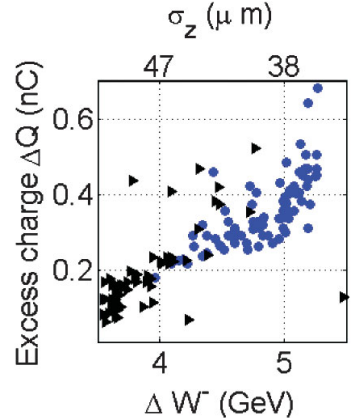

(b)
FIG. 2 (color). (a) Excess charge $\Delta Q$ as a function of energy loss $\Delta W^{-}$for four different beam propagation lengths in the plasma determined by changing $\epsilon_{n}$ by inserting foils in the path of the beam $\left(\sigma_{z}=40 \mu \mathrm{m}\right)$; (b) $\Delta Q$ as a function of $\Delta W^{-}$as the peak current of the beam is changed by changing $\sigma_{z}$. Estimated bunch length is given on the scale at the top [23] $\left(\epsilon_{n}=1.3 \epsilon_{N 0}\right.$, Foil 2). The variation in $\sigma_{z}$ is either from shot to shot variation or from a change in bunch compression setting (shorter bunch setting as blue circles, longer bunch setting as black triangles). Linear fit to the observed correlation between $\Delta W^{-}$and $\sigma_{z}$ is used to obtain the $\sigma_{z}$ scale in this figure. occurs due to a combination of a decreased $\sigma_{r}^{*}$ in the ion column and a longer propagation distance in the plasma.

Next, the beam propagation length is increased by increasing the beam current, i.e., by reducing $\sigma_{z}$ (while keeping the $\epsilon_{N}$ constant) to show that the increase in $\Delta Q$ is a result of a longer propagation length rather than a change in $\sigma_{r}^{*}$. As Fig. 2(b) shows, we once again see that as the $\Delta W^{-}$increases, so does $\Delta Q$. In this case, $\Delta Q$ is not affected by $\sigma_{r}^{*}$, since $\sigma_{r}^{*}$ does not depend on $\sigma_{z}$. Now the principal source of increase in both $\Delta W^{-}$and $\Delta Q$ is an increase of the interaction length and $E_{z}$, which occur as $\sigma_{z}$ is decreased. The first factor will increase $\Delta Q$ by increasing the number of envelope oscillations, while the latter will do the same by increasing net electric field $\sqrt{E_{r}^{2}+E_{z}^{2}}$ available for ionization of $\mathrm{Rb}^{+}$.

The reduction of $\langle T\rangle$ with increasing $\Delta Q$ can be visually observed on Fig. 3(a), which shows the energy spectra of electron bunches for the same data points that are depicted in Fig. 2(a). These spectra are arranged in order of increasing $\Delta Q$ with the divide between different beam emittance settings indicated. It can be observed that as $\Delta Q$ is increased, the $2 \%$ charge (energy gain) contour shows a slower increase than does the $98 \%$ charge (energy loss) contour. This results in a decrease in $\langle T\rangle$ values, which are plotted in Fig. 3(b) for these same data points. It is clear that $\langle T\rangle$ reduces from about 1 to 0.75 as the excess charge increases from 0.1 to $0.8 \mathrm{nC}$. The variation in $\langle T\rangle$ at a given value of $\Delta Q$ is probably due to subtle differences in the number of electrons in the tail of the electron bunch current profile, whereas the change in $\Delta Q$ at a given $\langle T\rangle$ is probably due to small changes to $\sigma_{r}^{*}$ that affect $E_{r}$ and, thus, the ionization rate of $\mathrm{Rb}^{+}$. Nevertheless, the general trend observed is that $\langle T\rangle$ decreases as $\Delta Q$ increases. This suggests that these $\mathrm{Rb}$ II electrons are beam loading the accelerating field and draining energy from the wake.

To show that beam loading by the Rb II electrons is the cause of the reduction of the transformer ratio, we have

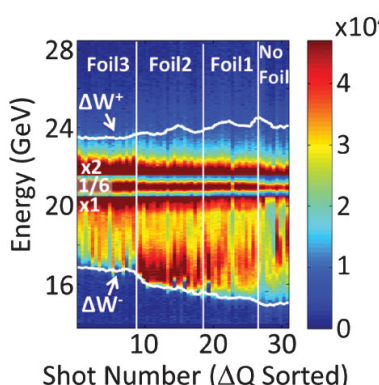

(a)

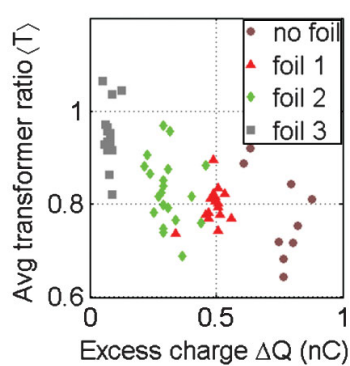

(b)
FIG. 3 (color). (a) Measured energy spectra of the same shots as displayed in Fig. 2(a). The values of energy loss $\Delta W^{-}$and gain $\Delta W^{+}$are shown as white lines and are displayed as a moving average of five shots. The numbers on the left hand side indicate the factors used on the data in the three primary energy ranges for better visibility, (b) the measured average transformer ratio $\langle T\rangle=$ $\Delta W^{+} / \Delta W^{-}$as a function of excess charge $\Delta Q$ leaving the plasma. 


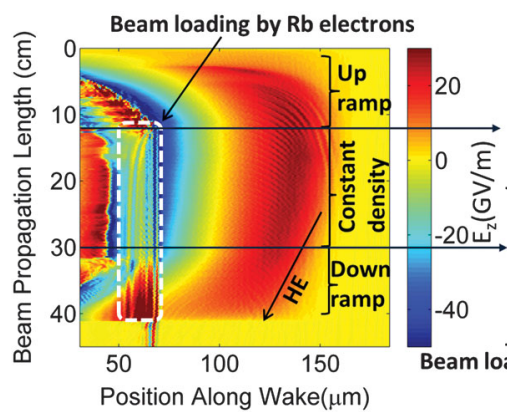

(a)

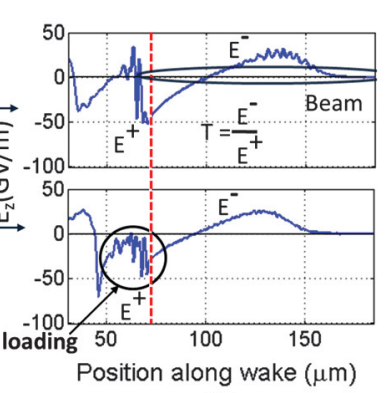

(b)
FIG. 4 (color). 2D cylindrical OSIRIS simulation of the experiment. (a) on axis electric field $\left(E_{z}\right)$ as a function of the beam propagation distance in the plasma. The dashed white rectangle indicates where the wake gets severely beam loaded by $\Delta Q$. The arrow marked HE shows the receding of the wake in the speed of light frame due to beam head erosion, (b) lineouts at two different locations [indicated by two horizontal arrows in (a)], the first one just at the end of the up ramp, and the second one after $30 \mathrm{~cm}$ of propagation in plasma at the end of the flat density region. The location used to measure $E^{+}$is shown by the red dashed line and is the location of a $2 \%$ beam charge at the end of the simulation. The effect of beam loading is evident in the bottom frame of (b) as a strong damping of the $E^{+}$field (circle).

carried out particle-in-cell simulations using the code OSIRIS [24] in 2D cylindrical coordinates. The simulation box has $480 \times 400$ square cells, each having a size of $0.05 k_{p}^{-1}$. Both $\mathrm{Rb}$ and Ar are included in the simulation. The ionization of $\mathrm{Rb}$ and $\mathrm{Ar}$ is modelled using the ADK formalism [22]. We use 16 particles per cell for each simulated species, and electrons from the creation of $\mathrm{Rb}^{+}$, $\mathrm{Rb}^{2+}$, and $\mathrm{Ar}^{+}$are tracked separately. The initial electron beam parameters and the plasma density profile are similar to those in the experiment.

We tracked the radius of one longitudinal slice of the beam (where the wakefield changes its sign, and therefore, electrons lose no energy) and, indeed, found that the RbII electrons at this location were produced each time this slice pinched to its smallest size $\sigma_{r}^{*}$, adding to the $\Delta Q$ in a discreet and distributed fashion. In Fig. 4(a), we show the variation of the on axis $E_{z}$ as a function of distance. One can clearly see the wavelength of the wake decreasing (increasing) in the up (down) ramp regions from $0-10 \mathrm{~cm}$ and $30-40 \mathrm{~cm}$, respectively. Also seen is the effect of near synchronous acceleration of excess charge (mostly Rb II electrons that overwhelm some Ar electrons injected in the up ramp) on $E_{z}$ from $10 \mathrm{~cm}$ onwards once the electron bunch enters the uniform $\mathrm{Rb}$ density region (the boxed region). Figure 4(b) shows two lineouts, one at $12 \mathrm{~cm}$, where the beam loading is not yet severe, and the other at $30 \mathrm{~cm}$, where the wake generated by the excess $\mathrm{Rb}$ II charge has nearly cancelled out the beam induced wake. This figure also shows that the value of $T$ decreases from its weakly unloaded value of $1.4\left(E^{+}=43 \mathrm{GV} / \mathrm{m}\right)$ at a distance of $12 \mathrm{~cm}$ to about $1\left(E^{+}=26 \mathrm{GV} / \mathrm{m}\right)$ at $30 \mathrm{~cm}$ where the flat $\mathrm{Rb}$ density region ends. The $\langle T\rangle$ seen by the beam electrons is $\sim 1$, which takes into account the effect of the ramps. In the experiment, $\langle T\rangle$ values as low as 0.8 are observed which may be due to somewhat heavier beam loading of the first bucket by the excess charge.

We note that although nearly $0.8 \mathrm{nC}$ of excess charge is produced in this experiment (mostly attributed to $\mathrm{Rb}$ II electrons), not all these electrons are necessarily being accelerated in the first bucket of the wake and, thus, do not contribute to beam loading. In the simulations, we observe that even though total $\Delta Q$ is up to $1 \mathrm{nC}$, only about $300 \mathrm{pC}$ of $\mathrm{Rb}$ II electrons are injected and accelerated in the first bucket and contribute to the beam loading and reduction of T. This latter amount is consistent with the estimate based on conservation of energy arguments given at the beginning of this Letter.

In conclusion, we have shown through experiments and simulation that distributed injection of electrons due to beam-induced ionization of $\mathrm{Rb}^{+}$can beam load the wake and reduce the accelerating field and the transformer ratio.

The work at UCLA was supported by DOE Grant No. DEFG02-92ER40727 and NSF Grant No. PHY-0936266. The simulations were carried out on the Hoffman cluster at UCLA. Work at SLAC was supported by Department of Energy Contract No. DE-AC02-76SF00515.

* Corresponding author. cjoshi@ucla.edu

[1] M. J. Hogan et al., New J. Phys. 12, 055030 (2010).

[2] P. Chen, J. M. Dawson, R. W. Huff, and T. Katsouleas, Phys. Rev. Lett. 54, 693 (1985).

[3] I. Blumenfeld et al., Nature (London) 445, 741 (2007).

[4] I. Blumenfeld, Ph.D. thesis, Stanford University, 2009.

[5] J. B. Rosenzweig, A. M. Cook, A. Scott, M. C. Thompson, and R. Yoder, Phys. Rev. Lett. 95, 195002 (2005).

[6] M. J. Hogan et al., Phys. Rev. Lett. 95, 054802 (2005).

[7] P. Muggli et al., Phys. Rev. Lett. 93, 014802 (2004).

[8] C. Joshi et al., Phys. Plasmas 9, 1845 (2002).

[9] R. Gholizadeh, T. Katsouleas, P. Muggli, C. Huang, and W. Mori, Phys. Rev. Lett. 104, 155001 (2010).

[10] T. Katsouleas, S. Wilks, P. Chen, J. Dawson, and J. Su, Part. Accel. 22, 81 (1987).

[11] E. Oz et al., Phys. Rev. Lett. 98, 084801 (2007).

[12] A. Pak, K. A. Marsh, S. F. Martins, W. Lu, W. B. Mori, and C. Joshi, Phys. Rev. Lett. 104, 025003 (2010).

[13] T. P. Rowlands-Rees et al., Phys. Rev. Lett. 100, 105005 (2008).

[14] C. McGuffey et al., Phys. Rev. Lett. 104, 025004 (2010).

[15] C. Clayton et al., Phys. Rev. Lett. 88, 154801 (2002).

[16] W. Lu, C. Huang, M. Zhou, W. B. Mori, and T. Katsouleas, Phys. Rev. Lett. 96, 165002 (2006); J. B. Rosenzweig, B. Breizman, T. Katsouleas, and J. J. Su, Phys. Rev. A 44, R6189 (1991).

[17] M. Tzoufras, W. Lu, F. Tsung, C. Huang, W. Mori, T. Katsouleas, J. Vieira, R. Fonseca, and L. Silva, Phys. Rev. Lett. 101, 145002 (2008). 
[18] D. Johnson et al., Phys. Rev. Lett. 97, 175003 (2006).

[19] I. Blumenfeld et al., Phys. Rev. ST Accel. Beams 13, 111301 (2010).

[20] A. Fisher and Z. Wu (private communication).

[21] P. Muggli, K. A. Marsh, S. Wang, C. E. Clayton, S. Lee, T. C. Katsouleas, and C. Joshi, IEEE Trans. Plasma Sci. 27, 791 (1999).
[22] D. L. Bruhwiler, D. A. Dimitrov, J. R. Cary, E. Esarey, W. Leemans, and R. E. Giacone, Phys. Plasmas 10, 2022 (2003).

[23] Z. Wu, A. S. Fisher, J. Goodfellow, M. Fuchs, D. Daranciang, M. Hogan, H. Loos, and A. Lindenberg, Rev. Sci. Instrum. 84, 022701 (2013).

[24] R. A. Fonseca et al., in Lecture Notes in Computer Science, (Springer, Berlin, 2002), Vol. 2331, pp. 342-351. 